\title{
LA ACCIÓN GLOBAL POR EL CLIMA Y LA IMPORTANCIA DE LOS JÓVENES EN EL MOVIMIENTO POR LA JUSTICIA CLIMÁTICA*
}

\author{
Global Climate Action and the Importance of Youth in the Climate Justice \\ Movement \\ Marcos de Armenteras Cabot \\ Universitat Rovira i Virgili \\ marcosdearmenteras@gmail.com
}

\begin{abstract}
Resumen:
A día de hoy, los jóvenes son una parte fundamental en la lucha por el clima y su voz está cada vez más presente dentro del movimiento. Las marchas más multitudinarias en los últimos años han sido lideradas por jóvenes de todo el mundo. El marco discursivo de las huelgas estudiantiles y los "Viernes por el Futuro" se fundamenta en la justicia intergeneracional y la necesidad de llevar a cabo políticas sobre el clima a largo plazo. En este artículo se analiza el movimiento por la justicia climática, y el ascendente rol de los jóvenes y de las demandas de justicia intergeneracional. En este artículo se analiza el movimiento por la justicia climática, y el ascendente rol de los jóvenes y de las demandas de justicia intergeneracional.
\end{abstract}

\section{Palabras clave:}

Movimiento por la justicia climática, justicia intergeneracional, cambio climático, Fridays for Future, acción global por el clima

\begin{abstract}
:
Today, young people are a fundamental part in the fight for the climate and their voice is increasingly present within the movement. The largest marches in recent years have been led by young people from around the world. The discursive framework of the student strikes and the "Fridays for the Future" is based on intergenerational justice and the need for longterm climate policies. This article looks at the climate justice movement, and the rising role of youth and intergenerational justice demands. This article looks at the climate justice movement, and the ascending role of youth and intergenerational justice demands.
\end{abstract}

\footnotetext{
Este trabajo ha sido desarrollado dentro del proyecto de investigación "Constitución climática global: gobernanza y Derecho en un contexto complejo", financiado por el Ministerio de Economía y Competitividad para el trienio 2017-2019 (proyecto DER201680011-P; IP: Jordi Jaria-Manzano y Susana Borràs Pentinat). El autor quiere agradecer los comentarios y sugerencias de dos revisores anónimos que ayudaron a mejorar este texto.
} 


\section{Keywords:}

Climate Justice Movement, Intergenerational Justice, Climate Change, Fridays for Future, Global Climate Action

Recibido: 02/11/2020

Aceptado: $28 / 12 / 2020$

\section{INTRODUCCIÓN}

El eco de las proclamas de los activistas se puede escuchar desde el exterior del recinto donde está concluyendo la vigésimo quinta Conferencia de las Partes (COP25) de la Convención Marco de Naciones Unidas para el Cambio Climático (CMNUCC) celebrada en Madrid durante las primeras semanas de diciembre de 2019'. Queremos justicia climática y la queremos ahora, reivindican ante las delegaciones reunidas para negociar asuntos pendientes del acuerdo firmado en París en el año 2015. Activistas y organizaciones no gubernamentales están dando la batalla para que se tomen medidas concretas frente al cambio climático. Medidas orientadas a mitigar los gases de efecto invernadero, plantear programas de adaptación, de transición energética y de mecanismos de compensación por los daños derivados del cambio climático. Además, demandan que este proceso sea guiado por estándares de justicia. Esto es, que las cargas derivadas del cambio climático y de los programas de mitigación y adaptación sean conmensuradas y asignadas según la capacidad de cada Estado.

Estos activistas y organizaciones son parte de un movimiento mucho más amplio que actúa globalmente, el movimiento por la justicia climática. Sin querer entrar en la discusión teórica sobre la definición de movimiento social, parto de la conceptualización que entiende que un movimiento social es un movimiento compuesto por redes informales de grupos y activistas sobre la base de una identidad colectiva compartida que se movilizan en torno a cuestiones conflictivas mediante la recurrencia frecuente a diversas formas de protesta (Diani, 1992: 3). Los movimientos sociales globales en este sentido serían redes supranacionales de actores que defienden sus causas como globales y organizan campañas de protesta que involucran a activistas de más de un Estado (Della Porta et al., 2006: 18). El movimiento por la justicia climática es un movimiento global de acción colectiva que aglutina a un amplio número de colectivos y activistas que exigen que las respuestas al cambio climático se den desde una perspectiva de justicia. Entre los grupos y activistas que forman parte de este movimiento destaca el rol de los jóvenes y menores, que enmarcan su lucha por el clima como una lucha por su futuro. Las huelgas estudiantiles por el clima y las manifestaciones alrededor del mundo muestran la fuerza de este colectivo.

En el año 2009, el Youth Climate Movement, se presentó en la cumbre de Copenhague bajo el lema, ¿Qué edad tendrás en 2050? Esta pregunta retórica encierra

${ }^{1}$ La vigésimo quinta Conferencia de las Partes de la CMNUCC se iba a celebrar en Santiago de Chile. Debido al estallido social contra las políticas del gobierno iniciadas en octubre de 2019, la COP25 se llevó a cabo en Madrid, España. 
dos problemas centrales del cambio climático: el impacto a largo plazo de lo daños al sistema climático, y el problema motivacional para tomar acciones debido a la distancia generacional entre aquellos que deben emprender medidas efectivas y aquellos que sufrirán las peores consecuencias. Es por ello, que los jóvenes y niños, que ven que su futuro depende de la toma de decisiones concretas y ambiciosas en el presente, han alzado su propia voz por la justicia climática.

En este artículo pretendo dar cuenta de la centralidad de las proclamas intergeneracionales en las protestas por el clima y el auge del movimiento juvenil por el clima. Para ello, analizaré en primer lugar por qué el cambio climático es un problema de justicia. En segundo lugar, daré cuenta de la evolución del movimiento por el clima y, seguidamente expondré la importancia del marco de justicia en el movimiento. Finalmente, daré cuenta de la importancia de los jóvenes en la acción global contra el clima y la centralidad del discurso de la justicia intergeneracional.

\section{EL CAMBIO CLIMÁTICO COMO PROBLEMA DE JUSTICIA}

El cambio del clima afecta y afectará de manera sustancial a todos los ecosistemas terrestres y marinos. El impacto en los ecosistemas se verá reflejado en una pérdida de la biodiversidad. Una de cada cinco especies de vertebrados, invertebrados y plantas están en riesgo de extinción debido al aumento de temperaturas (Otto, 2018:5). Las altas temperaturas provocarán largas temporadas de sequías y éstas llevarán a incendios más intensos, más frecuentes y más prolongados (Wehner et al., 2017: 232). Las olas de calor, los huracanes y las inundaciones serán más frecuentes e intensos. La temperatura en los océanos ha ido aumentando y lo seguirá haciendo lo que provoca su acidificación y la consecuente pérdida de biodiversidad. El nivel del mar aumenta anualmente y existen riesgos certeros de que la subida afecte a multitud de zonas costeras. El aumento de la temperatura también afecta a la criosfera, los científicos apuntan que en septiembre de 2007 el deshielo en el Ártico alcanzó el punto de no retorno (Serreze, 2007). Además, el deshielo del permafrost sería, si cabe, todavía más catastrófico. En el permafrost, capa de tierra cuya temperatura es inferior a la de congelación, se alberga una gran cantidad de $\mathrm{CO} 2$ y metano que si llegara a liberarse sería equivalente a la deforestación si ésta siguiera el ritmo actual, teniendo un efecto 2.5 veces mayor (Schuur y Abbott, 2011).

Además, de acuerdo con la investigación del Stockholm Resilience Centre, existen nueve límites planetarios interdependientes que son necesarios para el correcto funcionamiento de la tierra como sistema. Los nueve límites planetarios son el agotamiento del ozono estratosférico, la pérdida de la integridad de la biosfera, la contaminación química, el flujo de nitrógeno y fósforo a la biosfera y los océanos, la acidificación de los océanos, el consumo de agua dulce, el uso de tierra y la proporción de tierras cultivadas, la concentración de CO2 en la atmósfera, y la carga de aerosoles. Debido a su interdependencia, sobrepasar uno de ellos puede conducir a alterar irreversiblemente el funcionamiento de la tierra. De acuerdo con los últimos datos disponibles, cuatro de estos nueve límites ya se han superado (el cambio en el uso de tierras, la concentración de $\mathrm{CO} 2$, la pérdida de la integridad de la biosfera, y los flujos de nitrógeno y fósforo) y se estima que los otros cinco se sobrepasen en las 
próximas décadas, incluido el acceso a agua dulce, que se estima que sea un problema para 2050 (Stockholm Resilience Centre, 2009; Rockström et al., 2009).

El cambio climático es el resultado del calentamiento global producido por la acumulación excesiva de gases de efecto invernadero en la atmósfera. Una acumulación excesiva de gases de efecto invernadero causada por el actuar humano, principalmente por la quema de combustibles fósiles y la destrucción de sumideros naturales capaces de absorber dichos gases. Sin embargo, aunque el cambio climático es el resultado del actuar humano, no todas las sociedades, ni todos los individuos, dañan de igual forma el sistema climático, ni todas las sociedades, ni todos los individuos sufren o sufrirán sus consecuencias con la misma intensidad. Es por ello que es importante dar cuenta del cambio climático como un problema de justicia. Un problema vinculado con la distribución de cargas: la mitigación de gases de efecto invernadero, de transferencia de tecnología, de ayudas para programas de adaptación y de rendición de cuentas por responsabilidades por daño objetivo. También un problema de carácter intergeneracional que plantea qué modos de vida serán posibles en un mundo ecológicamente saturado (Riechmann, 2005: 98) habida cuenta de la necesidad de mantenerse dentro de los límites planetarios. En estos límites deberán vivir las futuras generaciones, pues cada año que se consumen más recursos de los que la tierra es capaz de regenerar, se está privando de recursos a las generaciones futuras.

De este modo, al trazar el origen de las emisiones de gases de efecto invernadero muchos estudios indican esta desigualdad. El informe que presentó la ONG Oxfam International del año 2015 evidencia la disparidad de emisiones entre individuos. Este informe se centra en analizar cómo las emisiones han sido y siguen siendo muy dispares entre individuos. Así, según el resultado de su investigación, aquellos individuos que se sitúan entre los 50\% más pobre de la población mundial generan el 10 $\%$ de las emisiones totales y aquellos situados en el $10 \%$ más rico generan más del $50 \%$. Además, la huella de carbono del $1 \%$ más rico es ciento setenta y cinco veces mayor que la del $10 \%$ más pobre (Gore, 2015). Este dato evidencia que la desigualdad económica es fundamental a la hora de analizar el origen y las responsabilidades vinculadas al cambio climático. Similarmente, el resultado del estudio publicado en el año 2014 por Richard Heede (2014) atribuye a 90 empresas un total de 70\% de las emisiones totales. En su última actualización (2019) el informe contabilizó 103 empresas que emitieron el $69,8 \%$ de las emisiones globales totales entre 1751 y 2017. Además, la mitad de estas emisiones se produjeron a partir de 1988, lo que implica que se han emitido más emisiones en los últimos 30 años que en los 237 anteriores (Heed, 2014: 7). En estos últimos treinta años, solamente 25 empresas han sido responsables del $51 \%$ total de las emisiones de gases de efecto invernadero entre 1988 y 2015 (Griffin, 2017:8). Estas empresas, conocidas como las carbon majors, son las mayores empresas dedicadas a la extracción (Heed, 2019) de combustibles fósiles -petróleo, gas y carbón- y a la producción de cemento. Este hecho revela que la mayoría de emisiones producidas por estas empresas se dio cuando la información sobre los impactos de los gases de efecto invernadero en el clima eran de sobra conocidos. 
Además de la disparidad de emisiones entre individuos y el rol que tienen algunas empresas en la gran parte de las emisiones, la disparidad también se evidencia entre Estados. Así, al tratar la disparidad de emisiones también es importante observar los impactos que tiene la actividad estatal en el cambio climático, ya sea en términos absolutos o per cápita. Es a partir de las emisiones estatales que se calcula la mitigación requerida por cada Estado. De este modo, la disparidad de las emisiones por Estado es evidente ya que tan sólo los Estados del G20 son responsables del 78\% de las emisiones totales (UNEP, 2019:16). En el mismo sentido, China, Estados Unidos, Unión Europea e India han emitido en la última década el 55\% de las emisiones totales, sin contar las emisiones vinculadas con el uso de la tierra. Si se tienen en cuenta las emisiones per cápita, además de los países productores de petróleo, destacan los países de la Unión Europea, Australia y Estados Unidos (World Bank, 2016).

La otra cara de la moneda es la de aquellos que experimentarán las peores consecuencias del cambio climático. Las futuras generaciones, aquellas personas que no han nacido pero que vivirán en el futuro, y los niños y jóvenes del presente, cuya expectativa de vida indica que vivirán hasta finales del siglo xxi, sufrirán las peores consecuencias del cambio climático. Principalmente porque las emisiones realizadas nos indican que la temperatura ascenderá en las próximas décadas y que la inacción nos sitúa en un aumento de temperatura por encima de los $3^{\circ} \mathrm{C}$ (UNEP, 2019). Con el paso del tiempo la vulnerabilidad se amplía porque a mayor temperatura, consecuencias más severas. La vulnerabilidad también depende del nivel de desarrollo de los Estados y también a su situación geográfica, y también de los factores de clase, origen, edad y condición física de los ciudadanos (Climate Vulnerability Monitor, 2017). De este modo, en general, las personas con discapacidad son más vulnerables al cambio brusco de temperaturas, tienen menor capacidad de reacción ante eventos extremos debido a su dependencia a recursos tecnológicos y debido a las necesidades de distinta índole que necesitan para cubrir sus necesidades (Jodoin et al., 2020). Los adultos mayores y los menores (Clark et al., 2020) también son más vulnerables a temperaturas extremas y situaciones de cambios drásticos de temperatura. Las comunidades indígenas también son especialmente vulnerables debido a su relación con el ambiente y los recursos naturales (Tsosie, 2007; Williams, 2012). Por último, los datos muestran que las mujeres pueden ser consideradas más vulnerables al cambio climático bajo circunstancias derivadas de catástrofes naturales (Denton, 2002; Arora-Jonsson, 2011).

Las desigualdades vinculadas con el cambio climático, tanto en sus causas como en sus consecuencias, sitúan el cambio climático como un problema de justicia de primer orden, tanto intrageneracional como intergeneracional. Es por ello que las medidas que se deben concretar para alcanzar la sostenibilidad y mantener el equilibrio del sistema climático debe determinarse a partir de un enfoque de justicia que tenga en cuenta la inequitativa distribución global y los impactos a largo plazo.

\section{EL MOVIMIENTO GLOBAL POR LA JUSTICIA CLIMÁTICA}

El cambio climático es, a día de hoy, la primera preocupación ciudadana a nivel global (Pew Research Centre, 2019). Hace más de treinta años, en el año 1988, el 
climatólogo James Hansen dio cuenta ante el Congreso de los Estados Unidos del grave peligro que suponía el cambio climático. A pesar de ello, las emisiones de gases de efecto invernadero no han dejado de aumentar. Igualmente los informes del Grupo Intergubernamental de Expertos sobre el Cambio Climático (IPCC), órgano científico creado por la Organización Mundial de Meteorología y el Programa de Naciones Unidas para el Medio Ambiente en 1988, llevan décadas avisando a la comunidad internacional de los peligros del cambio climático. La adopción del CMNUCC en 1992 y del Protocolo de Kioto en 1997 tampoco modificaron este curso de acción y la acumulación no ha dejado de aumentar. La dificultad de sumar a un acuerdo ambicioso a aquellos estados responsables de la gran parte de las emisiones ha llevado a los Estados a alcanzar compromisos insuficientes sin plantear una solución real y efectiva al cambio climático.

Frente a la inacción política y el continuo aumento de las emisiones, en los últimos treinta años la acción colectiva por el clima ha pasado de ser una proclama más de la luchas ecologistas, a plantearse como un movimiento autónomo que genera sus propias dinámicas y discursos, capaz de aglutinar a un buen número de actores de distintas procedencias. Así, un año más tarde del testimonio de Hansen y de la creación IPCC, en el 1989 se fundó la red de organizaciones no gubernamental para la lucha global contra el cambio climático, la Climate Action Network (CAN), con la pretensión de conectar a las diferentes organizaciones alrededor del mundo a través de una red única. Desde la creación de la red hasta la actualidad, el movimiento por el clima se ha transformado. De esta transformación es importante destacar dos elementos centrales. Uno es la estructura del movimiento en un marco a partir de la noción de "justicia climática" como núcleo auxológico del movimiento. Otro, es la progresiva expansión hacia un mayor número de colectivos que se visibiliza en la heterogeneidad del movimiento actual que va mucho más allá de colectivos u organizaciones ecologistas nacionales e internacionales.

La adopción de los "Principios de Bali por la Justicia Climática" en el año 2002 por una parte de las ONGs vinculadas con esta red supuso un avance para circunscribir la acción climática como un problema de justicia y no solo como un problema de orden tecnológico vinculado con proyectos de transición energética y desarrollo de tecnologías "renovables". Cinco años más tarde, en la COP13 celebrada en Bali, se formó definitivamente la red Ilamada "Climate Justice Now!", con la pretensión de unir a aquellas organizaciones que buscan articular soluciones al cambio climático a partir de premisas de justicia social, ecológica y de género (Climate Justice Now! Statement, 2008). Desde esta perspectiva, la lucha por el clima se traduciría no en una simple sustitución de fuentes de energías y la apuesta por un capitalismo verde, sino en una lucha por la democratización de la producción de alimentos y energía, en contraposición de la dependencia de los mercados globales de producción que naturalizan la injusta distribución actual y las relaciones de dominio y poder (De Lucia, 2009). Esto es, la lucha por la justicia climática, proyectaba el necesario cambio de modo de producción y consumo en el Norte Global y las élites del Sur Global.

Con estos antecedentes, es a partir de la COP15, celebrada en Copenhague en el año 2009, cuando la acción colectiva por el clima comenzó a alcanzar una mayor 
relevancia a escala global. La participación de organizaciones no gubernamentales ascendió un 35\% en comparación con la COP14, y los observadores pasaron de 4.000 a 13.000 en un solo año (Hadden, 2015: 19). Algunos factores determinantes para este cambio fueron el estreno del documental "An Inconvenient Truth" producido por Al Gore en el año 2006, la concesión del Premio Nobel de la Paz al IPCC y a Al Gore en 2007, o la publicación del cuarto informe del IPCC en el año 2007 en el que daba cuenta de las necesidades de mitigación para el año 2020. Estos factores, junto a la constatación de los impactos del cambio climático en hechos como la ola de calor sufrida en Europa en el año 2003, o el Huracán Katrina en el año 2005, pusieron en el punto de mira la necesidad de dar respuesta urgente al cambio climático (/dem).

Estos sucesos fueron precedidos por amplias olas de protestas convocadas por movimientos de resistencia global y altermundista. Los denominados "Días de la Acción Global", acciones de protestas descentralizadas y coordinadas por la red "Acción Global de los Pueblos", fueron el centro de gravedad de los movimientos sociales globales. Como detalla Matas Morell (2013: 84 - 85) la primera gran protesta descentralizada y global tuvo lugar ante la reunión ministerial de la OMC en Ginebra en 1998, que teniendo como epicentro la protesta en Ginebra, se realizaron protestas y manifestaciones en una treintena de estados (/dem). Entre otras, las protestas se replicaron en la posterior reunión ministerial de la OMC en Seattle en el año 1999, en los encuentros del Banco Mundial y el FMI en Washington y Praga en el año 2000, o ante la reunión del G-8 en Génova en el año 2001 (Della Porta et al:: 2006). Además, en el año 2001, se organizó la primera contracumbre de la reunión del Foro Económico Mundial, en Porto Alegre, donde se reunieron los activistas y organizaciones vinculadas con el movimiento por una globalización diferente (de Sousa Santos, 2005).

Para el caso que nos ocupa, el movimiento por la justicia climática volvió a mostrar su poder de convocatoria a escala global en el año 2014 en la "People's Climate March", que fue la mayor manifestación hasta la fecha llevada a cabo por el movimiento, reuniendo a 300.000 personas en la ciudad de Nueva York y más de 600.000 en otros 156 países $^{2}$. El año 2015 fue clave para el movimiento por la justicia global debido a que la firma del Acuerdo de París supuso un nuevo punto de inflexión. A pesar de que la ausencia de obligaciones de carácter normativo con respecto a la mitigación de gases de efecto invernadero frenó las aspiraciones de lograr un acuerdo ambicioso, el cambio de la estructura en la distribución de responsabilidades, pasando de un sistema en el que las reducciones de cada Estado Parte serían determinadas en el acuerdo (top-down), a un sistema en el que son los Estados Parte que determinan sus contribuciones esperadas (bottom-up), impactó notablemente en el movimiento por el clima. El Acuerdo de París, en su artículo 3 establece que las Partes deberán realizar y comunicar los esfuerzos ambiciosos para mantener el aumento de la temperatura media mundial muy por debajo de $2{ }^{\circ} \mathrm{C}$ con respecto a los niveles preindustriales, y proseguir los esfuerzos para limitar ese aumento de la temperatura a $1,5{ }^{\circ} \mathrm{C}$. Este cambio de estructura genera un cambio de escenario para la

${ }^{2}$ Se puede ver en la web de la BBC. Disponible en: https://www.bbc.com/news/scienceenvironment-29301969. Última visita el 20 de octubre de 2020. 
acción colectiva global. La acción colectiva global ya no sólo aparece como una protesta contra la comunidad internacional en su conjunto, sino que pueden ser demandadas dentro de los mismos Estados de forma directa para incidir en una mayor contribución del propio Estado a la mitigación del cambio climático.

\section{LA IMPORTANCIA DEL MARCO DE JUSTICIA}

Los efectos globales del cambio climático propician la pretensión de universalidad del movimiento. Las marchas por el clima son un llamamiento transversal para la luchar por la protección de la estabilidad climática que afecta, en mayor o menor medida, a la mayoría de la población mundial presente y futura. Este factor facilita que las demandas centrales del movimiento puedan superar la adscripción ideológica en el binario izquierda-derecha. Sin embargo, como ya he argumentado, el movimiento por el clima está enmarcado en la idea de justicia. Esto es, que las medidas encaminadas a mitigar, adaptar y atribuir las responsabilidades deben tener en cuenta quiénes tienen mayor capacidad para reducir su huella de carbono, quiénes pueden aportar más recursos para la adaptación y la transición ecológica, y dirimir quiénes son los responsables del cambio climático actual.

Según Della Porta y Parks (2014: 9), existen diferentes posturas dentro del movimiento por la justicia climática. Éstas podrían dividirse entre aquellos que plantean la impugnación total del sistema de producción y consumo que ha causado el cambio climático, y aquellos, que desde una perspectiva más pragmática -donde se situarían muchas ONG- que a pesar de ser críticos con el sistema económico, plantean modificaciones en él para alcanzar la mitigación y distribución de cargas. Si bien es cierto que existe esta heterogeneidad en el movimiento, considero que a día de hoy se trata de diferencias estratégicas que están vinculadas al rol que asume cada organización dentro del mismo movimiento, que diferencias sustantivas. Por ejemplo, la capacidad de influencia que tiene el "Climate Action Network", como red que aglutina a organizaciones de todo el mundo, en los procesos de negociación es muy diferente al rol que asumen movimientos de desobediencia civil y resistencia pasiva como "Extinction Rebellion". Esta diferencia está vinculada con las diferentes escalas en las que el movimiento actúa, y no con una diferencia ideológica sustantiva dentro del movimiento. La impugnación a los modos de producir, consumir y vivir que repercuten negativamente en el clima es central para el movimiento por la justicia climática, pues es incompatible plantear un cambio radical en las emisiones de efecto invernadero que pase por una reducción del consumo de energía y de materiales. En consecuencia, que el movimiento por el clima se desarrolle dentro del marco de la justicia climática implica que esté alejado de las tesis que plantean que la emergencia climática se resolverá solamente a través del desarrollo tecnológico, y una transferencia de tecnología que no ahonde en los problemas estructurales del sistema económico que nos ha llevado a esta emergencia.

El conocimiento científico no deja lugar a muchas interpretaciones. La reducción de emisiones llevada a cabo de forma ordenada y planificada, con efectos sociales y económicos de menor impacto, que habría sido posible acometer en los años ochenta y noventa, ya no es posible. En el último informe sobre la disparidad de las emisiones (UNEP, 2019) indica que para tener opciones reales de limitar el aumento de 
la temperatura global a $1,5^{\circ} \mathrm{C}$ deberíamos reducir nuestras emisiones a un $7,6 \%$ cada año hasta el 2030. Lo que significaría quintuplicar las reducciones actuales de emisiones de gases de efecto invernadero. Sin embargo, si la reducción hubiera comenzado en el año 2009, ahora mismo necesitaríamos una reducción de un 3,3\% anual, pero si la inacción política continúa hasta 2025, necesitaremos un $15,5 \%$ anual de reducción. Si las emisiones continúan y no se reducen llegaremos a un escenario en el año 2100 por encima de los $3^{\circ} \mathrm{C}$. A esta coyuntura, se suman los problemas derivados de la transición a energías no dependientes de combustibles fósiles. Principalmente porque las denominadas "energías renovables" son en realidad dispositivos no renovables, dependientes de minerales y otros recursos naturales limitados, que captan energías renovables, que deben ser fabricados, transportados e instalados, y que, además, producen energía eléctrica, cuando el consumo de electricidad representa menos del $20 \%$ del consumo energético mundial (IEA, 2019). En este sentido, las tesis ecomodernistas (Hickel, 2018) y post-ambientalistas (Kallis y Bliss, 2019), que pretenden dar salida a los problemas ecológicos a partir de cierto utopismo tecnológico, no se dan a día de hoy dentro del movimiento por la justicia climática.

Todo lo contrario. Es precisamente por cómo el mismo movimiento ha enmarcado sus demandas desde la COP13 en Bali, planteando un programa a partir de premisas como el consumo reducido, transferencias económicas de Norte a Sur, dejar de extraer combustibles fósiles, fomentar energías renovables, reconocimiento de los derechos de las comunidades indígenas sobre sus tierras, o fomentar sistemas de producción de alimentos sostenibles (Climate Justice Now! Statement, 2007), que el marco de referencia del movimiento está disociado de perspectivas productivistas que plantean tales alternativas tecnoutópicas que reproducen el mantra del "crecimiento verde". El framing en términos de justicia implica, justamente, que las soluciones pasen por mitigar y distribuir cargas vinculadas con el cambio climático, y que mitigar las emisiones no se traduzca en la transición a fuentes de energía no fósiles, sino que también suponga la reducción de forma equitativa del consumo de energía y materiales (Grubler et al, 2018; Hickel, 2019). Esto conlleva la incompatibilidad del paradigma del crecimiento económico en un escenario de reducción de emisiones de gases de efecto invernadero (Hickel y Kallis, 2019). En este sentido, la acción global por el clima plantea sus demandas desde una sólida evidencia científica y bajo premisas de justicia. Esta doble vertiente que nutre la acción impugna una manera de producir, consumir y vivir insostenible para dar paso a alternativas y modos de vida que no quiebren el sistema ecológico y climático. Ante este escenario, la pregunta planteada en el lema del "Youth Climate Movement" viene al caso: ¿Qué edad tendrás en 2050? Lo que implica preguntarnos qué modos de vida serán compatibles con el mundo que tendremos más allá del año 2050. La tensión entre los modos de vida que se proyecta en un mundo con escasez de recursos y programas de baja demanda energética chocan con lo que Wissen y Brand (2018) denominan "modos de vida imperiales". Según los autores estos modos de vida son la mayor dificultad para enfrentar los problemas ambientales. Éstos serían los patrones de consumo que llevan los individuos, principalmente del norte global -aunque también las clases altas del sur global-, que han normalizado un modo de vida basado en i) la apropiación ilimitada de recursos; ii) de un uso desproporcionado de los ecosistemas y sumideros mundiales y locales; y iii) de mano de obra barata de otros lugares, nor- 
malmente de la periferia de mundo capitalista. La existencia de esta tensión, entre los límites planetarios, los modos de producción y consumo, y las proyecciones de un futuro incierto, han activado a un buen número de movimientos juveniles a luchar por su futuro. En el 2050, cuando posiblemente se hayan traspasado los límites planetarios, los jóvenes que protestan hoy ya habrán llegado a la mediana edad.

Actualmente la voz de los jóvenes y menores, que observan un futuro incierto en el que su mundo será radicalmente diferente al que les ha precedido, es central en el movimiento por la justicia climática. El conflicto intergeneracional inherente al cambio climático, el cortoplacismo político y la inacción ante la emergencia climática, ha llevado a los jóvenes a ser una parte fundamental del movimiento por la justicia climática. En la última COP (2019), participaron 225 ONG juveniles, un 5,4\% del total (UNFCC, 2019). Si bien es cierto que la presencia de los jóvenes en las cumbres internacionales ha ido aumentando anualmente, la importancia de su presencia dentro del movimiento climático radica en el discurso que son capaces de enarbolar, fundamentado en que las decisiones de hoy determinarán su futuro.

\section{LOS JÓVENES EN EL MOVIMIENTO POR EL CLIMA}

En la Cumbre de Rio de 1992, Severn Suziki, una joven canadiense de doce años pronunció un discurso en nombre de la Environmental Children's Organization (ECO), una organización ecologista formada por jóvenes de doce y trece años. Suziki, con la pretensión de dar voz a su generación y a las generaciones venideras, plateó en su conciso discurso las injusticias de la distribución global de recursos y de las cargas vinculadas con los conflictos ambientales. El mensaje era claro, la lucha por su futuro y los de las generaciones por venir. Casi tres décadas más tarde, el 20 de agosto de 2018, tras una ola de calor y graves incendios en Suecia, una adolescente de 15 años Ilamada Greta Thunberg se manifestó ante del Parlamento Sueco para demandar acciones concretas por el clima. Esta joven inició una huelga escolar que duró tres semanas (hasta las elecciones legislativas) exigiendo a su Estado mayor ambición en las contribuciones nacionales establecidas en el Acuerdo de París. Su protesta siguió cada viernes. Los indicadores muestran que el tiempo que separan las palabras de Suziki y la primera protesta de Thunberg, la regresión ambiental no ha hecho más que empeorar. La cuestión es que de seguir en la línea actual de emisiones y consumo de energía y materiales, a una situación muy peligrosa en el año 2050.

La huelga iniciada por Thunberg inspiró las manifestaciones iniciadas en La Haya (Países Bajos) el 4 de Septiembre de 2018, en la que acudieron cinco estudiantes entre 15 y 17 años $^{3}$, en Berlín (Alemania) que desde el 14 de septiembre de 2018 se

\footnotetext{
${ }^{3}$ Se puede leer la nota del periódico neerlandés Algemeen Dagblad. Disponible en: https://www.ad.nl/den-haag/scholieren-spijbelen-voor-het-klimaat-boeit-onze-toekomst-jeiets a3cf7d $90 /$ ? referrer $=$ https $\% 3 \mathrm{~A} \% 2 \mathrm{~F} \% 2$ Fen.wikipedia.org\% $2 \mathrm{~F}$. Última visita el 24 de octubre de 2020.
} 
reunieron semanalmente frente al Bundestag ${ }^{4}$, y éstas desencadenaron protestas juveniles alrededor del mundo. La primera protesta estudiantil de gran impacto tuvo lugar en Australia, donde se manifestaron alrededor de 10.000 jóvenes entre 14 y 18 años en más de treinta ciudades ${ }^{5}$. Otras reivindicaciones de menor alcance se dieron en diferentes países, hasta que en diciembre de 2018, en la COP24 celebrada en Katowice (Polonia), Greta Thunberg, pronunció un discurso en nombre de Climate Justice Now! $!^{6}$. Su discurso, que se viralizó por las redes sociales, puso el foco en dos cuestiones: la injusticia intergeneracional vinculada con el cambio climático y que la emergencia climática no puede resolverse dentro de este sistema económico.

Tras el discurso de Thunberg, las manifestaciones juveniles se multiplicaron alrededor del mundo durante el primer trimestre del año 2019. Las manifestaciones Ilevadas a cabo en Bélgica, donde se manifestaron cada jueves desde el 10 de enero, y en Alemania, donde siguieron manifestando cada viernes, fueron particularmente numerosas. Las manifestaciones en todos los continentes llevaron a concretar una manifestación única el mismo día: el 15 de marzo de 2019. La marcha movilizó a más de 1,6 millones de personas alrededor del mundo (Wahlström et al., 2019: 6), y alrededor de 1 millón de estudiantes alrededor del mundo secundaron la huelga por el clima, en más de 125 países y 2.000 protestas ${ }^{7}$. Esta acción se repitió el 24 de mayo del mismo año con un resultado similar: secundado en 110 países y alrededor de un millón de manifestantes ${ }^{8}$. En septiembre de 2019, y a dos meses de la COP25 de Santiago de Chile, finalmente celebrada en Madrid, se organizaron manifestaciones masivas de forma global. La denominada "Global Week for Future" que tuvo lugar entre el 20 y el 27 de septiembre de 2019. Durante la "Global Week for the Future" se manifestaron alrededor de siete millones de personas alrededor del mundo, en más de 4.500 manifestaciones en 150 países. La primera gran manifestación, celebrada el 20 de septiembre reunión a más de 4 millones de personas alrededor del mundo ${ }^{9}$. La segunda manifestación, celebrada el viernes 27 de septiembre, reunión a

\footnotetext{
4 Se puede leer la nota del periódico alemán Der Tagesspiegel . Disponible en: https://www.tagesspiegel.de/politik/proteste-vor-dem-bundestag-schueler-demonstrieren-inberlin-gegen-den-klimawandel/23761834.html. Última visita el 24 de octubre de 2020.

${ }^{5}$ Se puede leer la nota del periódico estadounidense The New York Times (Australia). Disponible en: https://www.nytimes.com/2018/11/30/world/australia/student-strike-climatechange.html. Última visita el 24 de octubre de 2020.

6 Se puede leer en la página de youtube de Fridays for future. Disponible en: https://youtu.be/CcOp_17WqAk. Última visita el 24 de octubre de 2020.

7 Se puede leer la nota del periódico The Guardian. Disponible en: https://www.theguardian.com/environment/live/2019/mar/15/climate-strikes-2019-live-latestclimate-change-global-warming. Última visita el 24 de octubre de 2020.

8 Se puede leer la nota del periódico The Guardian. Disponible en https://www.theguardian.com/environment/2019/may/24/schoolchildren-go-on-strike-acrossworld-over-climate-crisis. Última visita el 24 de octubre de 2020.

${ }^{9}$ Se puede leer en el medio digital Vox. Disponible e https://www.vox.com/energy-andenvironment/2019/9/20/20876143/climate-strike-2019-september-20-crowd-estimate. Última visita el 26 de octubre de 2020 .
} 
más de 2 millones de personas en diferentes localizaciones ${ }^{10}$. Finalmente, el viernes 6 de diciembre de 2019, durante la celebración de la COP25, se llevó a cabo una manifestación multitudinaria en Madrid, donde acudieron más de medio millón de personas ${ }^{11}$.

La capacidad movilizadora del movimiento juvenil por el clima no tiene precedentes en el movimiento. Además, esta capacidad de movilización, no es óbice para la inclusión de un discurso muy crítico con el sistema que ha llevado a la tierra hasta esta situación de emergencia. Esta crítica se puede observar tanto en la politización de la vida cotidiana, como el rechazo de Thunberg a viajar en avión o su preferencia de dietas vegetarianas o veganas, o en la influencia del movimiento en otros aspectos de la política ambiental, como la reciente protesta ante la nueva Política Agrícola Común (PAC) ${ }^{12}$. Todo ello, se debe sumar a la clave en perspectiva de justicia intergeneracional que emerge del discurso por la justicia climática y que es capaz de aglutinar a gran parte de una generación. Los jóvenes se sienten interpelados por un futuro incierto y los graves problemas ambientales que deberán afrontar en las décadas por venir.

En este sentido el movimiento por el clima aglutina a los movimientos vinculados con el ecologismo e interpela de manera directa a generaciones de jóvenes que observan cómo aquel futuro que no iba a llegar está cada día más cerca y cómo la inacción ante el cambio climático afectará directamente a sus vidas en las próximas décadas. El marco de justicia del movimiento por el clima no sólo observa desde el punto de vista espacial, sino también desde el temporal. El movimiento por el clima se plantea desde el marco de justicia entre coetáneos, pero también con aquellos que vivirán en la tierra las próximas décadas. De este modo, superar los límites planetarios y no incidir en la mitigación de las emisiones de gases de efecto invernadero supone asumir que las generaciones de jóvenes de hoy y las futuras generaciones asumirán toda la carga de la inacción: escasez hídrica, aumento de las temperaturas, acidificación de los océanos, incendios, desertificación, etcétera. ¿Qué edad tendrás en 2050?, pregunta el movimiento por la justicia climática. La respuesta interpela a aquellas generaciones que estarán vivas y sufrirán las consecuencias más allá del 2050. Esta interpelación plantea la importancia central de asumir la responsabilidad hoy para poder evitar las peores consecuencias del cambio climático en un futuro cercano.

La clave generacional que propulsa los jóvenes actualmente es clave para entender el futuro del movimiento por el clima. La inacción llevará a mayor movilización y aumentará la capacidad de aglutinar del movimiento y agregar a subsiguientes gru-

10 Se puede leer la nota del periódico The Guardian. Disponible en: https://www.theguardian.com/environment/2019/sep/27/climate-crisis-6-million-people-joinlatest-wave-of-worldwide-protests. Última visita el 26 de octubre de 2020.

11 Se puede leer la nota del periódico The Guardian. Disponible en: https://www.theguardian.com/environment/2019/dec/09/cop25-climate-summit-whathappened-during-first-week

12 Se puede leer en la web de France 24. Disponible en: https://www.france24.com/en/europe/20201025-thousands-of-climate-activists-appeal-tobrussels-to-withdraw-farm-policy. Última visita el 26 de octubre de 2020. 
pos generacionales. Actualmente, la voz de los jóvenes tiene mucha fuerza en el movimiento por la justicia climática y no sólo es escuchada por parte del movimiento, sino que ha involucrado a muchos jóvenes en esta lucha entorno a la necesidad de dar soluciones justas al cambio climático.

\section{CONCLUSIONES}

Pour ce qui est de l'avenir, il ne s'agit pas de le prévoir, mais de le rendre possible ${ }^{13}$. Esta cita de Antoine de Saint-Exupéry encabeza el informe especial del IPCC del año 2018 (IPCC Special Report, 2018). Hacer el futuro posible es sin duda la pretensión del movimiento por la justicia climática. Como he argumentado, el movimiento por el clima se fundamenta en un sólido conocimiento científico aportado desde hace décadas por el IPCC, y sus planteamientos se enmarcan en demandas de justicia. Así, que el futuro sea posible para las generaciones de jóvenes y las futuras generaciones va de la mano de dar soluciones justas en la actualidad, que la distribución de cargas sea equitativa, y que se tenga una mirada a largo plazo. Para ello, el movimiento de justicia climática plantea una impugnación al sistema y a los modos de vida que han llevado a la tierra a esta situación límite.

El planteamiento desde una perspectiva de justicia entre generaciones pone sobre la mesa que estos modos de producción, consumo y vida, no sólo son injustos entre las generaciones presentes, también lo son con las generaciones futuras. Este factor propicia a que las generaciones de jóvenes de muchos países de todo el mundo se sientan interpelados por los problemas intergeneracionales que plantea el cambio climático. Como he señalado anteriormente, si la reducción de emisiones hubiera comenzado en el año 2009, se habría requerido una reducción anual del 3,3\% de las emisiones, si hubiera comenzando en el año 2019 una reducción del 7,6\% hasta 2030, y de comenzar en 2025, las reducciones deberían ser entorno al 15,5 anual (UNEP, 2019). Cada año que los estados dejen de actuar, el esfuerzo de las generaciones futuras será mayor. Así, el esfuerzo en reducción de emisiones deberá ser más ambicioso por cada año de inacción política. Omitir la reducción urgente y necesaria de emisiones no sólo llevará a la reducción desordenada en el futuro, sino también a la superación de los límites establecidos por la comunidad científica. Es por ello que los jóvenes de hoy, que ya están observando el aumento de las temperaturas año tras año, plantean la necesidad de actuar con urgencia hoy, para que la tierra en el futuro cercano siga siendo habitable.

Es por estos motivos que el movimiento por la justicia climática interpela a la juventud, pues no sólo ven que los modos de vida que ha llevado una parte del mundo hasta la actualidad es insostenible, sino que este modo de vida pone en peligro su futuro y el de las generaciones que les sucederán. Así, aunque el movimiento por la justicia climática es amplio y heterogéneo, y está compuesto por una gran cantidad de grupos de activistas y organizaciones de todo el mundo, atender al rol y la voz de los jóvenes es determinante para comprender el crecimiento, la expansión y el alcance que tiene actualmente el movimiento por la justicia climática.

13 "En cuanto al futuro, no se trata de predecirlo, sino de hacerlo posible", Antoine de SaintExupéry Citadelle, Paris, Gallimard, 1948. 
BIBLIOGRAFÍA

ARORA-JONSSON, S. (2011). "Virtue and vulnerability: Discourses on women, gender and climate change". Global Environmental Change, 21(2)

CLARK et al. (2020). "A future for the world's children? A WHO-UNICEF-Lancet Commission". World Health Organization. [Disponible en: https://www.thelancet.com/journals/lancet/article/PIIS0140-6736(19)325401/fulltext. Última visita el 20 de febrero de 2020]

CLIMATE JUSTICE NOW! STATEMENT (2008). [Disponible en: http://www.carbontradewatch.org/take-action-archive/climate-justice-nowstatement-4.html. Última visita el 25 de octubre de 2020]

CLIMATE VULNERABLE FORUM (2017). "Second Climate Vulnerability Monitor: a guide to the cold calculus of a hot planet". [Disponible en: https://daraint.org/wp-content/uploads/2012/09/CVM2ndEd-FrontMatter.pdf. Última visita 25 de octubre de 2020]

DE LUCIA, V., (2009). "Hegemony and Climate Justice: A Critical Analysis", en BÖHM, S., y DABHI, S. (Eds.) Upsetting the Offset: The Political Economy of Carbon Markets, London, MayFly Books.

DE SOUSA SANTOS, B. (2005). Foro Social Mundial: Manual de uso. Icaria

DELLA PORTA, D. y DIANI, M. (1999). Social Movements: An Introduction, Blackwell

DELLA PORTA, D., ANDRETTA, M., KOLAROVA, M. y MOSCA, L. (2006). Globalization from Below: Transnational Activists and Protest Networks

DELLA PORTA, D., y PARKS, L. (2014). "Framing Processes in the Climate Movement: From Climate Change to Climate Justice" en DIETZ, M., y GARRELTS, H. (Eds.), Routledge handbook of climate change movements. Routledge

DIANI, M. (1992). "The Concept of Social Movement", Sociological Review 40(1): 1 25.

FATMA DENTON, S. (2002). "Climate change vulnerability, impacts, and adaptation: Why does gender matter?" Gender \& Development, 10 (2)

GRIFFIN, P. (2017). "The Carbon Majors Database". CDP Carbon Majors Report. [Disponible en: https://www.cdp.net/en/articles/media/new-report-shows-just100-companies-are-source-of-over-70-of-emissions.última visita el 20 de octubre de 2020]. 
GORE, T. (2015). "La desigualdad extrema de las emisiones de carbono: por qué el acuerdo sobre el clima de París debe anteponer los intereses de las personas más pobres, vulnerables y que generan menos emisiones de carbono", Oxfam International. [Disponible en: https://oi-files-d8-prod.s3.eu-west2.amazonaws.com/s3fs-public/file_attachments/mb-extreme-carbon-inequality021215-es.pdf. Última visita el 20 de octubre de 2020].

GRUBLER, A., WILSON, C., BENTO, N. et al. (2018). A low energy demand scenario for meeting the $1.5^{\circ} \mathrm{C}$ target and sustainable development goals without negative emission technologies, Nature Energy, 3, 515-527.

HADDEN, (2015). Networks in Contention: The Divisive Politics of Climate Change. Cambridge University Press

HEEDE, R. (2013). "Carbon Majors: Accounting for carbon and methane emissions 1854-2010 Methods \& Results Report", commissioned by Climate Justice Programme (Sydney) \& Greenpeace International (Amsterdam), Climate Mitigation Services, Snowmass, CO. [Disponible en http://climateaccountability.org/pdf/MRR\%209.1\%20Apr14R.pdf. Última visita el 20 de octubre de 2020]

HEEDE, R. (2019). "Carbon Majors: Updating activity data, adding entities, \& calculating emissions: A Training Manual", Climate Accountability Institute, Snowmass, Colorado. [Disponible en: https://climateaccountability.org/pdf/TrainingManual\%20CAI\%2030Sep19lores .pdf. Última visita el 20 de enero de 2020. Última visita el 20 de octubre de 2020].

HICKEL, J. (2018). "The Magical Thinking of Ecomodernism". Climate \& Capitalism. [Disponible en: https://climateandcapitalism.com/2018/05/16/the-magicalthinking-of-ecomodernism/. Última visita el 20 de octubre de 2020]

HICKEL, J. (2019). "Degrowth: a theory of radical abundance". Real-World Economics Review, Issue N. 87

HICKEL, J., y KALLIS, G. (2019). "Is Green Growth Possible?", New Political Economy, 25(7576), 1-18

IEA - INTERNATIONAL ENERGY AGENCY. (2019), World Energy Outlook 2019, IEA, Paris https://www.iea.org/reports/world-energy-outlook-2019

IPCC (2018). "Global Warming 1.5 Special Report". [Disponible en: https://www.ipcc.ch/sr15/. Última visita el 25 de octubre de 2020]

JODOIN, S., ANANTHAMOORTHY, N., y LOFTS, K. (2020). "A Disability Rights Approach to Climate Governance", Ecology Law Quarterly, Vol. 47:1 
KALLIS, G. y BLISS, .(2019). "Post-environmentalism: origins and evolution of a strange idea", Journal of Political Ecology, Vol. 26, N.1, $466-485$

MATAS MORELL, A. (2013). "Algunas lecciones emancipadoras del movimiento antiglobalización. El caso de la "Acción Global de los Pueblos»". Oxímora Revista Internacional de Ética y Política, N. 2

OTTO, S.P., (2018). "Adaptation, speciation and extinction in the Anthropocene". Proceedings of the Royal Society B: Biological Sciences, 285: 20182047

PEW RESEARCH CENTER. (2019). "Climate Change Still Seen as the Top Global Threat, but Cyberattacks a Rising Concern". [Disponible en: https://www.pewglobal.org/wp-content/uploads/sites/2/2019/02/Pew-ResearchCenter_Global-Threats-2018-Report_2019-02-10.pdf. Última visita el 23 de octubre de 2020]

RIECHMANN, J., (2005). “¿Cómo cambiar hacia sociedades sostenibles? Reflexiones sobre biomímesis y autolimitación", Isegoría, 32, 95 - 118

ROCKSTRÖM, J., STEFFEN, W., NOONE, K. et al. (2009). "A safe operating space for humanity". Nature 461, 472-475

SCHUUR, E. y ABBOTT, B. (2011) "High risk of permafrost thaw", Nature, Comment

SERREZE, M. (2007). "Arctic Sea Ice Minimum Shatters All-time Record Low, Report Scientists". University of Colorado at Boulder. ScienceDaily. ScienceDaily, 21 September 2007. [Disponible en: www.sciencedaily.com/releases/2007/09/070920160226.html. Última visita el 23 de octubre de 2020]

STOCKHOLM RESILIENCE CENTRE. (2009). "The Nine Planetary Boundaries" [Disponible en: http://www.stockholmresilience.org/research/planetaryboundaries/planetary-boundaries/about-the-research/the-nine-planetaryboundaries.html. Último acceso 20 de octubre de 2020]

TSOSIE, R. (2007). "Indigenous People and Environmental Justice: The Impact of Climate Change". University of Colorado Law Review, 78, 1625

UNFCC - Statistics on Participation and in-session engagement. (2019). [Disponible en: https://unfccc.int/process-and-meetings/parties-non-party-stakeholders/nonparty-stakeholders/statistics-on-non-party-stakeholders/statistics-onparticipation-and-in-session-engagement. Última visita el 20 de octubre de 2020]

UNEP - UNITED NATIONS ENVIRONMENT PROGRAMME. (2019). "Emissions Gap Report", Nairobi. [Disponible en: https://wedocs.unep.org/bitstream/handle/20.500.11822/30797/EGR2019.pdf?s equence $=1$ \&isAllowed $=y$. Última visita 20 de octubre de 2020] 
WAHLSTRÖM, M., KOCYBA, P., DE VYDT, M., y DE MOOR, J. (Eds.) (2019). Protest for a future: Composition, mobilization and motives of the participants in Fridays For Future climate protests on 15 March, 2019 in 13 European cities. [Disponible en: https://eprints.keele.ac.uk/6571/7/20190709_Protest\%20for\%20a\%20future_G CS\%20Descriptive\%20Report.pdf. Última visita el 25 de octubre de 2020]

WEHNER, M.F., ARNOLD, J.R., KNUTSON, T., KUNKEL, K.E., y LEGRANDE, A.N. (2017). "Droughts, floods, and wildfires". En WUEBBLES, D.J., FAHEY D.W., HIBBARD, K.A., DOKKEN D.J., STEWART, B.C., y MAYCOCK T.K. (eds.). Climate Science Special Report. Fourth National Climate Assessment, Volume I. U.S. Global Change Research Program, Washington, DC, USA

WILLIAMS, J. (2012). "The impact of climate change on indigenous people - the implications for the cultural, spiritual, economic and legal rights of indigenous people", The International Journal of Human Rights, Volume 16, Issue 4

WISSEN, M. y BRAND, U. (2018). "Imperial Mode of Living". Krisis, Journal for Contemporary Philosophy, Issue 2: Marx from the Margins

WORLD BANK. (2016). The World Bank Data [Disponible en: https://data.worldbank.org/indicator/EN.ATM.CO2E.PC. Última visita el 20 de octubre de 2020] 